\title{
Correlation for Solubilities of Water in Compressed Nonpolar Gases
}

\author{
Hironori Miyahara*, Satoru Suzuta* and Yasuhiko Arai*
}

\begin{abstract}
By using a perturbed-hard-sphere equation of state, a useful procedure has been proposed for estimating the equilibrium moisture content in compressed gases resulting from some processes.

The solubility of water, expressed in mole fraction, in compressed nitrogen, argon, carbon dioxide, methane, and ethane can be well correlated in the range from $25^{\circ}$ to $100^{\circ} \mathrm{C}$ and up to $100 \mathrm{~atm}$ by introducing two characteristic binary constants into the mixing rules for $a_{m}$ and $c_{m}$.
\end{abstract}

\section{Introduction}

In recent years, much attention has been paid to the correlation of water solubility in compressed nonpolar gases. The solubility information is very important to evaluate the equlibrium moisture content in compressed gases, such as inert gases and light hydrocarbon gases resulting from some processes.

De Santis et al. ${ }^{1)}$ have shown that the RedlichKwong equation of state is useful to correlate the solubility of water in compressed $n$-butane gas by adjusting the constant $a$ reflecting the intermolecular attraction. De Santis and Marrelli2) have adopted a perturbed-hard-sphere equation of state (the repulsion term is given by Carnahan and Starling's expression ${ }^{3}$ ) and the attraction term is approximated by that of the Redlich-Kwong equation of state) to correlate the solubility of water in compressed air. More recently, Luks et al. ${ }^{4)}$ have found that the modified Redlich-Kwong equation of state of Heidemann and Prausnitz ${ }^{5}$ ) is useful for correlating the water content of combustion gases saturated with moisture.

In a previous paper ${ }^{6}$ ), the perturbed-hard-sphere equation of state proposed by Nakamura et al. ${ }^{7}$ ) has been found to be successful to represent both the $p-v-T$ relations and the second and third virial coefficients for water vapor. In the present study, the equation of state is applied to correlate the solubility of water in several compressed nonpolar gases.

\section{Equation of State}

The perturbed-hard-sphere equation of state proposed by Nakamura et al. ${ }^{7)}$ has the following form:

\footnotetext{
Received Aug. 31, 1981.

* Department of Chemical Engineering, Kyushu University (6-10-1, Hakozaki, Higashi-ku, Fukuoka 812)
}

$$
z=\frac{p v}{R T}=\frac{1+\xi+\xi^{2}-\xi^{3}}{(1-\xi)^{3}}-\frac{a}{R T(v+c)}
$$

where

$$
\xi=\frac{b}{4 v}
$$

Parameter $b$ reflects the hard-core size of the molecule and parameter $a$ reflects the strength of attractive forces. Parameter $c$ is empirically introduced to successfully represent the $p-v-T$ relations of strongly polar components. In a previous study $^{6}$ ), all parameters, $a, b$, and $c$, have been found dependent on temperature to accurately represent both the $p-v-T$ relations and the virial coefficients of water vapor. The parameters $a, b$, and $c$ expressed in the reduced forms are as follows:

$$
\begin{aligned}
& \frac{a}{R T_{c} v_{c}}=\sum_{i=0}^{3} a^{(i)} T_{r^{-i}} \\
& \frac{b}{v_{c}}=\sum_{i=0}^{4} b^{(i)} T_{r}{ }^{i} \\
& \frac{c}{v_{c}}=\sum_{i=0}^{6} c^{(i)} T_{r}{ }^{-i}
\end{aligned}
$$

The coefficients in Eqs. (3) to (5) for water vapor, presented in Table 1, have been determined in a previous study by use of the $p-v-T$ relations and the second and third virial coefficients. These coefficients are found here to be very successful in calculating the molar volume, fugacity coefficients, and enthalpies of water vapor, including saturated regions, by comparing the calculated results with experimental data ${ }^{8)}$.

The values of coefficients $a^{(i)}, b^{(i)}$, and $c^{(i)}$ of several nonpolar gases have been determined and they are shown in Table 1 . In the present determination, the $p-v-T \mathrm{data}^{8) \sim 11)}$ are firstly used. That is, the deviations between calculated and experimental values for $(z-1) v$ have been minimized. Then, the second and third virial coefficient data $^{12}$ ) are adopted to fit more precisely 
Table 1 Parameters for a Perturbed-Hard-Sphere Equation of State

\begin{tabular}{|c|c|c|c|c|c|c|}
\hline & Nitrogen & Argon & Carbon Dioxide & Methane & Ethane & Water \\
\hline Range & $\begin{array}{c}166-450 \mathrm{~K} \\
\text { up to } 100 \mathrm{~atm}\end{array}$ & $\begin{array}{c}130-600 \mathrm{~K} \\
\text { up to } 5,000 \mathrm{~atm}\end{array}$ & $\begin{array}{c}223-423 \mathrm{~K} \\
\text { up to } 200 \mathrm{~atm}\end{array}$ & $\begin{array}{c}273-373 \mathrm{~K} \\
\text { up to } 400 \mathrm{~atm}\end{array}$ & $\begin{array}{c}240-500 \mathrm{~K} \\
\text { up to } 700 \mathrm{~atm}\end{array}$ & $\begin{array}{c}300-870 \mathrm{~K} \\
\text { up to } 290 \mathrm{~atm}\end{array}$ \\
\hline $\begin{array}{l}a^{(0)} \\
a^{(1)} \\
a^{(2)} \\
a^{(3)}\end{array}$ & $\begin{array}{c}-2.5015486 \\
31.60246 \\
-91.091705 \\
87.313229\end{array}$ & $\begin{array}{r}2.3859247 \\
-3.9871658 \\
5.3221259 \\
-2.0173053\end{array}$ & $\begin{array}{l}0.65108680 \\
0.78109055 \\
0.32887296 \\
0\end{array}$ & $\begin{array}{l}1.3726333 \\
0.27564089 \\
-0.26691900 \\
0\end{array}$ & $\begin{array}{l}0.95659009 \\
0.23236945 \\
0.50280264 \\
0\end{array}$ & $\begin{array}{r}1.966 \\
-1.684 \\
0.8393 \\
0.6166\end{array}$ \\
\hline $\begin{array}{l}b^{(0)} \\
b^{(1)} \\
b^{(2)} \\
b^{(3)} \\
b^{(4)}\end{array}$ & $\begin{array}{r}5.4596275 \\
-6.8822815 \\
3.5163760 \\
-0.79404039 \\
0.066921382\end{array}$ & $\begin{array}{l}0.85156221 \\
-0.43356124 \\
0.18390608 \\
-0.023563303 \\
0\end{array}$ & $\begin{array}{l}0.51562805 \\
0.13875773 \\
-0.14895046 \\
0 \\
0\end{array}$ & $\begin{array}{l}-0.18641436 \\
0.70192292 \\
-0.17216026 \\
0 \\
0\end{array}$ & $\begin{array}{l}1.0850499 \\
-0.83272574 \\
0.26287190 \\
0 \\
0\end{array}$ & $\begin{array}{l}0.4946 \\
-0.07109 \\
0.01738 \\
0 \\
0\end{array}$ \\
\hline $\begin{array}{l}c^{(0)} \\
c^{(1)} \\
c^{(2)} \\
c^{(3)} \\
c^{(4)} \\
c^{(5)} \\
c^{(6)}\end{array}$ & $\begin{array}{c}-3.2315364 \\
39.383658 \\
-171.10222 \\
328.22313 \\
-234.60092 \\
0 \\
0\end{array}$ & $\begin{array}{l}0.0064893423 \\
0.043762737 \\
-0.071726863 \\
0.034354091 \\
0 \\
0 \\
0\end{array}$ & $\begin{array}{l}0.10154717 \\
0.34050308 \\
-0.20892004 \\
0 \\
0 \\
0 \\
0\end{array}$ & $\begin{array}{l}1.6055528 \\
-8.8322325 \\
15.300526 \\
-8.1621628 \\
0 \\
0 \\
0\end{array}$ & $\begin{array}{l}-0.0994264 \\
0.64133174 \\
-0.38075839 \\
0 \\
0 \\
0 \\
0\end{array}$ & $\begin{array}{r}61.089 \\
-376.372 \\
933.266 \\
-1187.312 \\
814.760 \\
-283.650 \\
38.268\end{array}$ \\
\hline $\begin{array}{l}T_{c} \\
v_{c}\end{array}$ & $\begin{array}{r}125.95 \\
90.07\end{array}$ & $\begin{array}{r}151.0 \\
75.2\end{array}$ & $\begin{array}{r}304.2 \\
94.0\end{array}$ & $\begin{array}{r}190.65 \\
99.16\end{array}$ & $\begin{array}{l}305.4 \\
148.0\end{array}$ & $\begin{array}{r}647.3 \\
57.1\end{array}$ \\
\hline
\end{tabular}

in the low density region.

In order to apply Eq. (1) to binary mixtures, the following mixing rules are adopted.

$$
\begin{aligned}
& a_{m}=y_{1}^{2} a_{1}+y_{2}{ }^{2} a_{2}+2 y_{1} y_{2}\left(1-k_{12}\right)\left(a_{1} a_{2}\right)^{1 / 2} \\
& b_{m}=y_{1} b_{1}+y_{2} b_{2} \\
& c_{m}=y_{1}{ }^{2} c_{1}+y_{2}{ }^{2} c_{2}+y_{1} y_{2}\left(1-l_{12}\right)\left(c_{1}+c_{2}\right)
\end{aligned}
$$

where $k_{12}$ and $l_{12}$ are the characteristic binary constants. The mixing rule for $c_{m}$ is proposed here to make it applicable even though the values of parameters $c_{1}$ and $c_{2}$ are opposite in sign.

\section{Thermodynamics of Water Solubility}

The relation of vapor-liquid equilibrium for water is expressed by

$$
\phi_{2}{ }^{V} y_{2} p=\gamma_{2}{ }^{L} x_{2} p_{2}{ }^{\circ} \phi_{2}{ }^{\circ V} \exp \left[\frac{v_{2}{ }^{\circ L}\left(p-p_{2}{ }^{\circ}\right)}{R T}\right]
$$

where $p_{2}{ }^{\circ}$ and $v_{2}{ }^{\circ L}$ are, respectively, the saturation vapor pressure and the saturated liquid molar volume of pure water at a given temperature. They can readily be calculated from the empirical equations of Prausnitz et al. ${ }^{13)}$. The solubility of any gas in liquid water is assumed to be negligible when compared to unity; i.e. $x_{2}=1$. Accordingly, the liquid phase activity coefficient of water $\gamma_{2}{ }^{L}$ can be taken to be unity without introducing an appreciable error. This assumption may not be suitable for the carbon dioxide-water system.

However, this crude approximation is adopted for all gases to develop a practical estimating procedure which in many cases may be sufficiently accurate for engineering purposes. The fugacity coefficient $\phi_{2}{ }^{V}$ can be derived as follows from Eqs. (1) and (6) to (8) according to the thermodynamic standard procedure.

$$
\begin{aligned}
\ln \phi_{2} V & =\frac{4 \xi-3 \xi^{2}}{(1-\xi)^{2}}+\frac{b_{2}}{b_{m}}\left[\frac{4 \xi-2 \xi^{2}}{(1-\xi)^{3}}\right]+\frac{a_{m}}{R T\left(v^{V}+c_{m}\right)} \\
& +\frac{a_{m}+2\left[y_{1}\left(1-k_{12}\right)\left(a_{1} a_{2}\right)^{1 / 2}+y_{2} a_{2}\right]}{c_{m} R T} \\
& \times \ln \left(\frac{v^{V}}{v^{V}+c_{m}}\right) \\
& -\frac{a_{m}\left[y_{1}\left(1-l_{12}\right)\left(c_{1}+c_{2}\right)+2 y_{2} c_{2}\right]}{R T} \\
& \times\left[\frac{1}{c_{m}^{2}} \ln \left(\frac{v^{V}}{v^{V}+c_{m}}\right)+\frac{1}{c_{m}\left(v^{V}+c_{m}\right)}\right]-\ln z
\end{aligned}
$$

The fugacity coefficient of saturated vapor for pure water $\phi_{2}{ }^{\circ V}$ can readily be obtained by letting $y_{2}=$ 1.

\section{Correlation of Water Solubility}

The values of two characteristic binary constants $k_{12}$ and $l_{12}$ are necessary to calculate the water solubility expressed in mole fraction $y_{2}$ by use of Eq. (9). From Eqs. (1), (6) and (7) the second virial cross coefficient $B_{12}$ can be derived as

Table 2 Characteristic Binary Constants $\left(25-100^{\circ} \mathrm{C}\right)$

\begin{tabular}{l|c|c|c|cc}
\hline $\begin{array}{c}\text { Binary System } \\
(1)-(2)\end{array}$ & $k_{12}$ & $l_{12}$ & $\begin{array}{c}\text { Max. } \\
\text { Press. } \\
(\text { atm })\end{array}$ & Max. & Avg. \\
\hline Nitrogen-Water & 0.648 & 0.835 & 100 & 10.1 & 3.1 \\
Argon-Water & 0.687 & 1.079 & 92 & 12.1 & 1.6 \\
Carbon Dioxide- & 0.455 & 0.992 & 51 & 6.6 & 3.2 \\
Water & 0.657 & 1.040 & 92 & 3.0 & 1.3 \\
Methane-Water & 0.638 & 1.056 & 36 & 3.4 & 1.8 \\
Ethane-Water & 0.63 & \\
\hline
\end{tabular}

* Error $(\%)=100 \times\left|y_{2}^{\mathrm{exp}}-y_{2}^{\mathrm{calc}}\right| / y_{2}^{\mathrm{exp}}$, Avg. $=\frac{1}{N} \sum_{i=1}^{\mathrm{N}}(\text { Error })_{i}$ $N=$ No. of data points 


$$
B_{12}=\frac{1}{2}\left[\left(b_{1}+b_{2}\right)-\frac{2\left(1-k_{12}\right)\left(a_{1} a_{2}\right)^{1 / 2}}{R T}\right]
$$

Therefore, the values of $k_{12}$ can be estimated when experimental data for $B_{12}$ are available. On the basis of the literature values ${ }^{\mathbf{1 4}), 15}$, the values of $k_{12}$ for nonpolar gas-water binary mixtures have been estimated. The values of $l_{12}$, on the other hand, have been determined here to give best fit to experimental $y_{2}$. The values of $k_{12}$ and $l_{12}$ thus obtained are presented in Table 2. The procedure used for calculating $y_{2}$ is shown in Fig. 1. Typical representations for comparison between calculated and experimental solubilities of water in compressed gases are illustrated in Figs. 2 to 4. As shown in these figures, a good agreement is shown for wide ranges of temperatures and pressures.

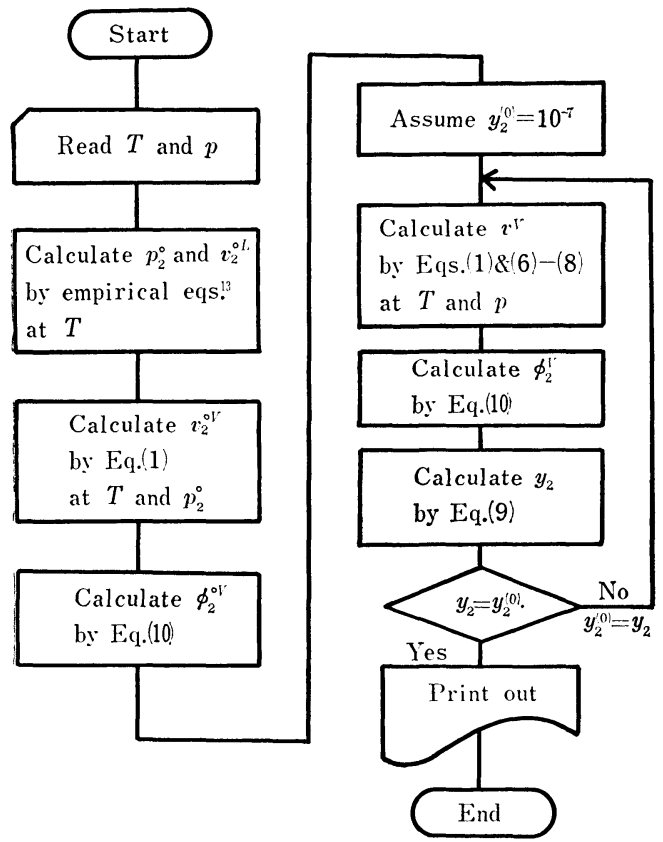

Fig. 1 Calculation Procedure for Water Solubility in Compressed Gases



Fig. 2 Solubilities of Water in Compressed Methane

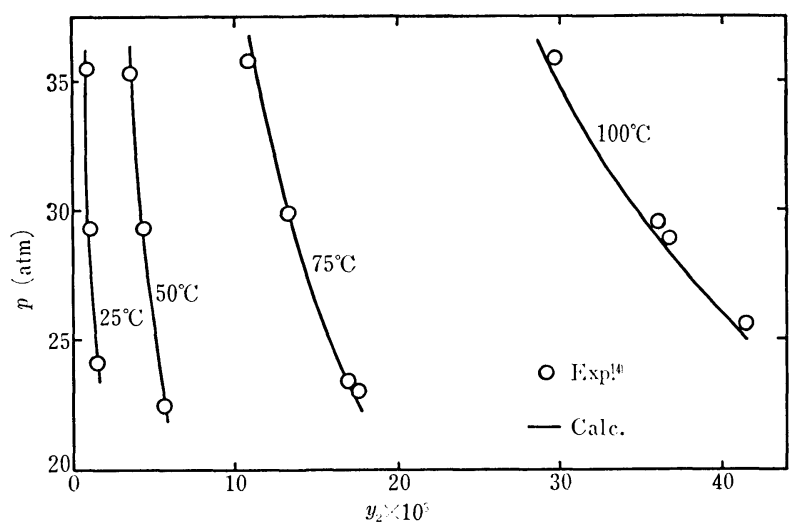

Fig. 3 Solubilities of Water in Compressed Ethane

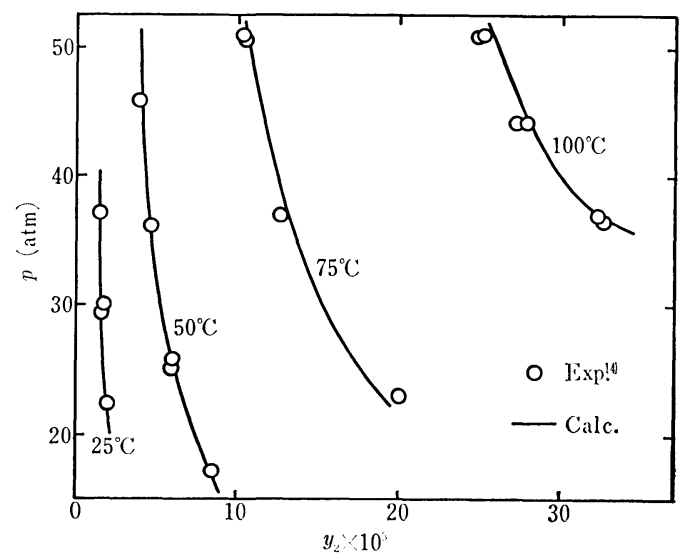

Fig. 4 Solubilities of Water in Compressed Carbon Dioxide

\section{Conclusion}

The perturbed-hard-sphere equation of state has been found to be successful to correlate the solubility of water in compressed gases such as nitrogen, argon, carbon dioxide, methane, and ethane in the range from $25^{\circ}$ to $100^{\circ} \mathrm{C}$ and up to 100 atm. In order to obtain a good correlation, two characteristic binary constants are required for the mixing rules for $a_{m}$ and $c_{m}$.

\section{Nomenclature}

$a, b, c=$ coefficients in Eq. (1)

$a^{(i)}, b^{(i)}, c^{(i)}=$ coefficients in Eqs. (3)-(5)

$B_{12}=$ second virial cross coefficient $\left(\mathrm{cm}^{3} / \mathrm{mol}\right)$

$k_{12}=$ characteristic binary constant

$l_{12}=$ characteristic binary constant (-)

$p=$ total pressure (atm)

$p_{2}^{\circ}=$ saturation vapor pressure of pure water $(\mathrm{atm})$

$R=$ gas constant $\quad\left(\mathrm{cm}^{3} \cdot \mathrm{atm} / \mathrm{mol} \cdot \mathrm{K}\right)$

$T=$ absolute temperature $\quad(\mathrm{K})$

$v=$ molar volume $\quad\left(\mathrm{cm}^{3} / \mathrm{mol}\right)$

$x=$ liquid phase mole fraction (-)

$y_{1}=$ vapor phase mole fraction of nonpolar gas 
$y_{2}=$ vapor phase mole fraction of water (=water solubility in compressed gas)

$z=$ compressibility factor $(=p v / R T)$

$\gamma=$ activity coefficient

$\xi=$ reduced density $(=b / 4 v)$

$\phi=$ fugacity coefficient

\section{Superscripts}

$L=$ liquid phase

$V=$ vapor phase

$\circ=$ pure state

\section{Subscripts}

$c=$ critical property

$m=$ mixture

$r=$ reduced property $\left(T_{r}=T / T_{c}\right)$

$1=$ nonpolar gas

$2=$ water

\section{References}

1) De Santis, R., Breedveld, G. J. F., Prausnitz, J. M., Ind. Eng. Chem. Process Des. Dev., 13, 374 (1974).

2) De Santis, R., Marrelli, L., Canadian J. Chem. Eng., 55, 712 (1977)

3) Carnahan, N. F., Starling, K. E., J. Chem. Phys.,
51, 635 (1969).

4) Luks, K. D., Kohn, J. P., Banchero, J. T., Ind. Eng. Chem. Process Des. Dev., 18, 564 (1979).

5) Heidemann, R. A., Prausnitz, J. M., Ind. Eng. Chem. Process Des. Dev., 16, 375 (1977).

6) Nishida, N., Ohba, M., Arai, Y., Fluid Phase Equilibria, 4, 303 (1980).

7) Nakamura, R., Breedveld, G. J. F., Prausnitz, J. M., Ind. Eng. Chem. Process Des. Dev., 15, 557 (1976).

8) Canjar, L. N., Manning, F. S., "Thermodynamic Properties and Reduced Correlation for Gases", (1967), Gulf Publ. Co., Houston Texas.

9) Din, F., "Thermodynamic Functions of Gases", Vol. 1, 2, (1956), Butterworths Scient. Publ., London.

10) Douslin, D. R., Harrison, R. H., Moore, R. T., McCullough, J.P., J. Chem. Eng. Data, 9, 358(1964).

11) Douslin, D. R., Harrison, R. H., J. Chem. Thermodynamics, 5, 491 (1973).

12) Dymond, J. H., Smith, E. B., "The Virial Coefficients of Gases", (1969), Clarendon Press, Oxford.

13) Prausnitz, J. M., Eckert, C. A., Orye, R. V., O'Connell, J. P., "Computer Calculations for Multicomponent Vapor-Liquid Equilibria", (1967), PrenticeHall Inc., Englewood Cliffs, N. J.

14) Coan, C. R., King, A. D. Jr., J. Am. Chem. Soc., 93, 1857 (1971).

15) Rigby, M., Prausnitz, J. M., J. Phys. Chem., 72, 330 (1968). 


\title{
無極性加圧気体に対する水の溶解度の相関
}

\author{
宮原 弘法*, 鈴田＼cjkstart聡*, 荒井 康彦*
}

加圧気体中の平衡水分含有量に関する知見は, 石油化学関連 プロセスにおける設計および運転にとって，きわめて重要であ る。一例をあげると，水分量がある程度以上になると加圧ガス との間に気体水和物（ガスハイドレート）を形成し, 安定した 供給や輸送が困難となることが知られている。一方，極性の著 しく強い水分子と無極性の不活性気体や低級炭化水素気体から なる混合物の特性を良好に表現する状態方程式の開発の観点か らも，興味を集めている(1),2),4),5)。

温度 $T$, 全圧 $p$ における加圧気体中の水分量をモル分率 $y_{2}$ で表現すると, 熱力学関係式より次式で与えられる。

$$
y_{2}=\frac{\gamma_{2}{ }^{L} x_{2} p_{2}{ }^{\circ} \phi_{2}{ }^{\circ} V}{\phi_{2} V p} \exp \left[\frac{v_{2}{ }^{\circ} L\left(p-p_{2}{ }^{\circ}\right)}{R T}\right]
$$

ここで温度 $T$ における純水の飽和液体モル体積 $v_{2}{ }^{\circ}{ }^{2} お$ よび飽 和蒸気圧 $p_{2}{ }^{\circ}$ は, 広く用いられている実験式 ${ }^{13)} に よ り$ 求める ことができる。いま水相に対する気体の溶解度が無視できると すれば，水相に打ける水のモル分率 $x_{2}$ を 1 と近似でき，活量 係数 $\gamma_{2}{ }^{L}$ む 1 とおくことができる。この仮定は, 溶解度の比 較的大きい二酸化炭素については厳密なものとはいえないが, $y_{2}$ が満足に 相関できたので，計算上の簡便さから，すべての 気体について $x_{2}=1 ， \gamma_{2}{ }^{L}=1$ の仮定を用いた。したがって，気 相中の水のフガシチー係数 $\phi_{2} V$ および純水の気相フガシチー 係数 $\phi_{2}{ }^{\circ} \mathrm{V}$ が与兄られる $y_{2}$ 寸なわち水分量が計算できる。 フガシチー係数は, 適当な状態方程式より導出できるが, ここ

* 九州大学工学部化学機械工学科 (812 福岡市東区箱崎 6-10-1)
では前報6)における次式を採用した。

$$
\frac{p v}{R T}=\frac{1+\xi+\xi^{2}-\xi^{3}}{(1-\xi)^{3}}-\frac{a}{R T(v+c)}
$$

ここで, $\xi=b /(4 v)$ であり， 3 個のパラメーター $a, b, c$ を含 む剛体球流体 ${ }^{3}$ を基準にした拱動型状態方程式である。この型 の状態方程式は, Nakamura らうの提案によるものであるが， 著者らの研究6)により水蒸気の $p-v-T$ および第 2 , 第 3 ビリ アル係数を満足に 表現し得ることが 示されている。本研究で は, 前報6) と同様に $p-v-T$ データ ${ }^{8)}{ }^{11)}$ およびビリアル係数デ 一タ12)を用い，窒素，アルゴン，二酸化炭素，メタンおよびェ タンのパラメーター $a, b, c$ を温度の関数として決定し, Teble 1 に示した。状態方程式を気体混合物に適用するためには，ハ ラメーターの混合則が必要とされる (本文中, Eqs. (6)〜 (8) 参 照)。パラメーター $a, b$ については通常用いられているもの を採用したが，cについては成分間で符号が異なる場合もあ り，新たな混合則を用いた。フガシチー係数 $\phi_{\mathbf{2}}{ }^{V}$ および $\phi_{\mathbf{2}}{ }^{\circ} V$ を求めることにより（本文中, Eq. (10)参照) 水分量が算出さ れるが，必要とされる 2 個の 2 成分系特性因子のらちパラメー ター $a$ に含まれるものは交差第 2 ビリアル係数データ ${ }^{14)}{ }^{15)}$ 上 り決定した。パラメーター $c$ に含まれるものは， $y_{2}$ の計算値 が実測值と最もよく合うように試行によって決定し，それぞれ Table 2 に示した。加圧気体に対する水の溶解度の計算手順 を Fig. 1 に示し, 計算値と実測值の比較を Figs. 2〜4 亿示 すが, 両者の一致は満足すべきものであり, 工学的計算法とし て有用と思われる。

\section{Keywords}

Compressed nonpolar gas, Lower hydrocarbon, Solubility, Water 